\title{
FORTSCHRITTE \\ DER CHEMIE ORGANISCHER NATURSTOFFE
}

EINE SAMMLUNG VON ZUSAMMENFASSENDEN BERICHTEN

UNTER MITWIRKUNG VON

A. BUTENANDT - W. N. HAWORTH · F. KŐGL · E. SPÃTH BERLIN

BIRMINGHAM

UTRECHT

WIEN

HERAUSGEGEBEN VON

\section{ZECHMEISTER}

PÉCS

\section{ZWEITER BAND}

BEARBEITET VON

Y. ASAHINA - CH. DHÉRÉ - K. FREUDENBERG

C. R. HARINGTON - E. L. HIRST - F: KUFFNER - H. RUDY

E. SPATH · G. TÓTH - L. ZECHMEISTER - G. ZEMPLÉN

MIT 24 ABBILDUNGEN IM TEXT

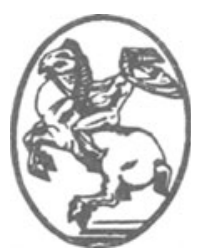

WIEN

VERLAG VON JULIUS SPRINGER

1939 
ISBN-13: 978-3-7091-7185-1

DOI: 10.1007/978-3-7091-7184-4

e-ISBN-13: 978-3-7091-7184-4

ALLE RECHTE, INSBESONDERE DAS DER UBERSETZUNG

IN FREMDE SPRACHEN, VORBEHALTEN

COPYRIGHT I939 BY JULIUS SPRINGER IN VIENNA

Softcover reprint of the hardcover 1st edition 1939 


\section{Inhaltsverzeichnis.}

Lignin. Von Professor Dr. K. Fre UdEnBERG, Universität Heidelberg... I

I. Einleitung $\ldots \ldots \ldots \ldots \ldots \ldots \ldots \ldots \ldots \ldots \ldots \ldots \ldots \ldots \ldots \ldots$ I

2. Verwandte Pflanzenstoffe $\ldots \ldots \ldots \ldots \ldots \ldots \ldots \ldots \ldots \ldots \ldots \ldots \ldots \ldots \ldots \ldots \ldots, 2$

3. Zustand und Bindung des Lignins im Holze................... 3

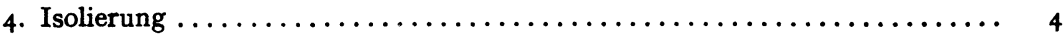

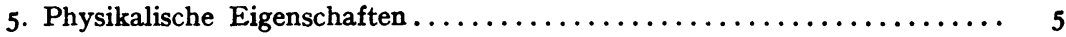

6. Analytische Unterlagen .............................. 6

7. Lignin als Derivat des Phenylpropans ..................... 7

8. Uber die Konstitution des Fichtenlignins.................... 7

9. Funktionelle Derivate $\ldots \ldots \ldots \ldots \ldots \ldots \ldots \ldots \ldots \ldots \ldots \ldots \ldots \ldots$ ro

Io. Substitutionsprodukte $\ldots \ldots \ldots \ldots \ldots \ldots \ldots \ldots \ldots \ldots \ldots \ldots \ldots \ldots \ldots$ ro

II. Unmittelbarer Abbau............................. II

12. Abbau nach Aufschluß mit Alkali........................ I2

13. Abbau nach Aufschluß mit Bisulfit. Ligninsulfonsäure........... I4

14. Abbau nach Aufschluß mit Thioglykolsäure .................. I6

15. Abbau nach Aufschluß mit Alkoholen und Mineralsäuren........... I6

16. Abbau nach Aufschluß mit Hydrazin, sowie mit Kalium in Ammoniak. I8

17. Dehydro-diisoeugenol und sëine Umwandlungsprodukte als Modelle ..... I8

18. Andere Modelle .............................. I9

19. Coniferylalkohol als Modell......................... 20

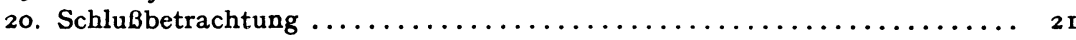

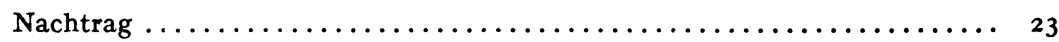

Literaturverzeichnis............................. 24

Flechtenstoffe. Von Professor Dr. Y. Asahina, Universităt Tokyo .... 27

A. Verbindungen der Fettreihe $\ldots \ldots \ldots \ldots \ldots \ldots \ldots \ldots \ldots \ldots \ldots \ldots \ldots \ldots \ldots \ldots \ldots$

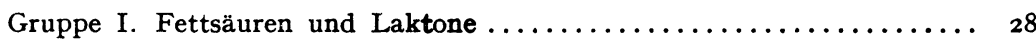

I. Protolichesterinsäuren und ihre Derivate $\ldots \ldots \ldots \ldots \ldots \ldots \ldots, 28$

2. Nephromopsinsäure.......................... 3о

3. Nephrosterinsäure und Nephrosteransäure $\ldots \ldots \ldots \ldots \ldots \ldots \ldots$, 30

4. Caperatsäure.............................. $3^{\text {I }}$

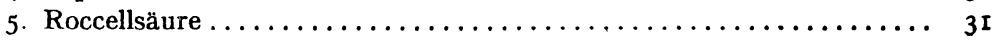

Gruppe II. Neutrale, gegen Alkali indifferente Substanzen $\ldots \ldots \ldots \ldots \ldots, 32$

Zeorin und Leukotylin ............................ $3^{2}$

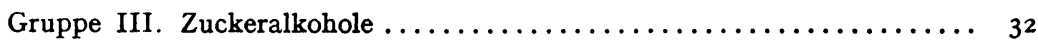

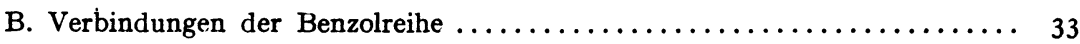

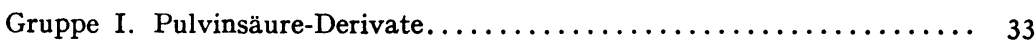

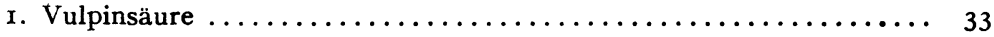

2. Pinastrinsäure $\ldots \ldots \ldots \ldots \ldots \ldots \ldots \ldots \ldots \ldots \ldots \ldots \ldots \ldots \ldots \ldots \ldots \ldots, 33$

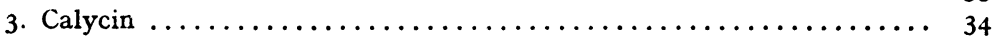




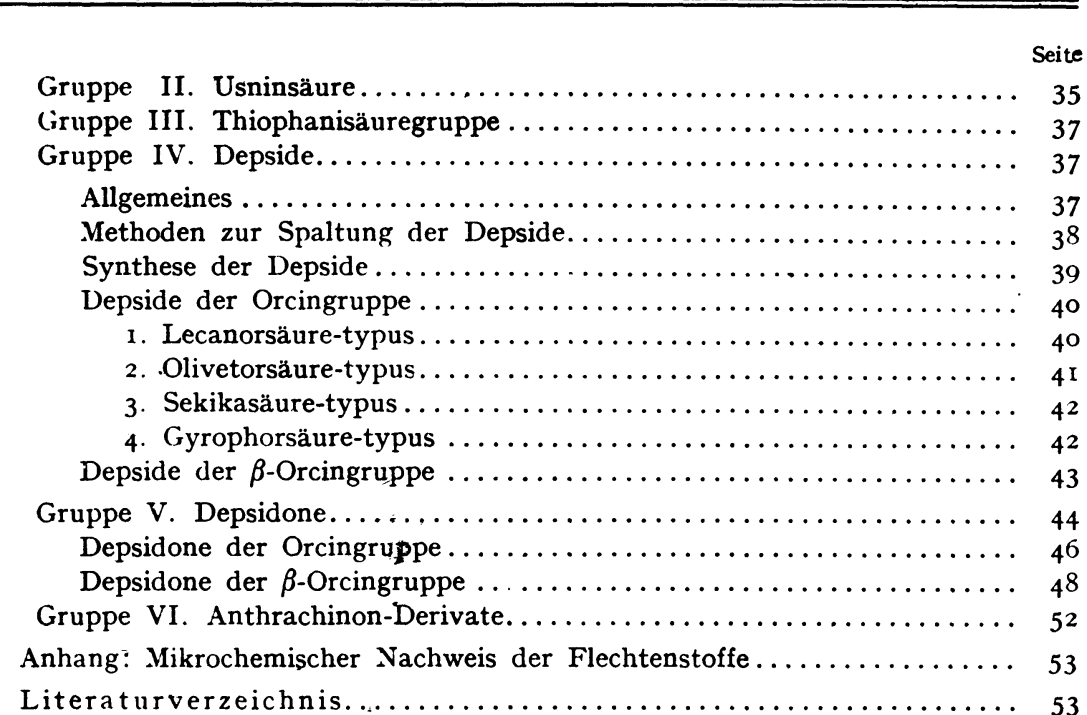

Flavine. Mit besonderer Berücksichtigung des Lactoflavins. Von Privatdozent Dr. H. RuDY, Lniversität Erlangen .............. 6r

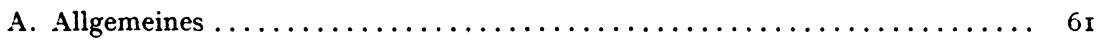

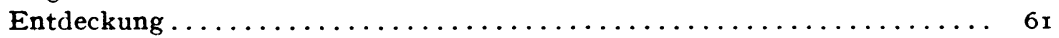

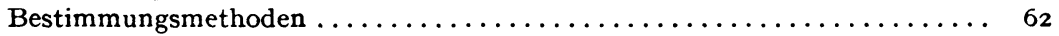

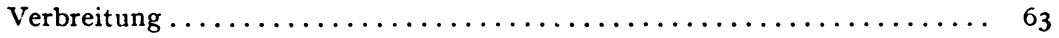

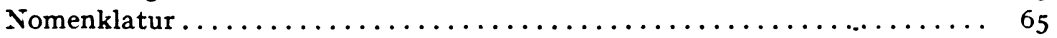

Schmelzpunkt, Farbe, Spektrum, Fluoreszenz ............... 66

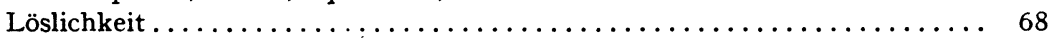

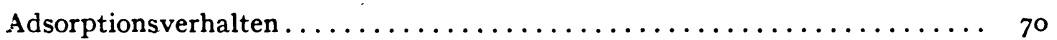

Verhalten gegen Mineralsäuren und Oxydationsmittel $\ldots \ldots \ldots \ldots \ldots \ldots$ 70

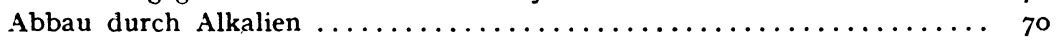

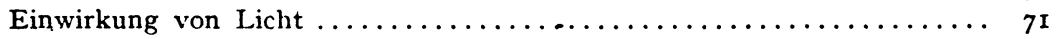

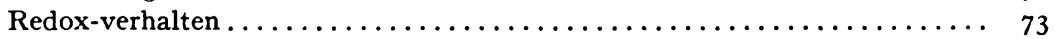

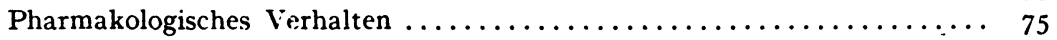

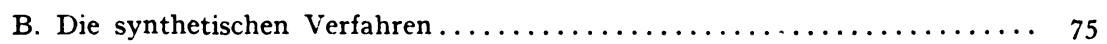

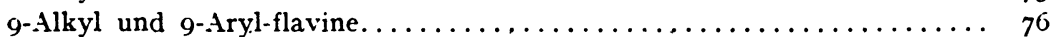

Flavin-9-alkylcarbonsäuren. . . . . . . . . . . . . . . . . . . .

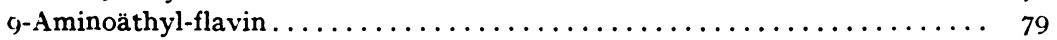

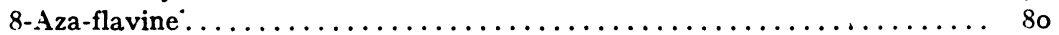

9-Oxyalkyl- und 9-Polyoxyalkyl-flavine ................... $8_{\mathrm{I}}$

Synthese der Oxy- und Polyoxy-flavine, einschließlich Lactoflavin ..... 82

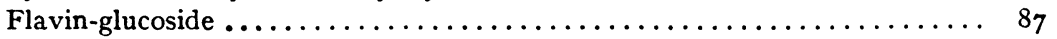

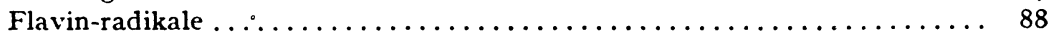

C. Flavine als wasserstoff-übertragende Cofermente $\ldots \ldots \ldots \ldots \ldots \ldots \ldots$ go

Flavin-phosphorsäuren $\ldots \ldots \ldots \ldots \ldots \ldots \ldots \ldots \ldots \ldots \ldots \ldots \ldots \ldots \ldots$ gr

Lactoflavin-5' -phosphorsäure (Alloxazin-mono-nucleotid) ......... 9r

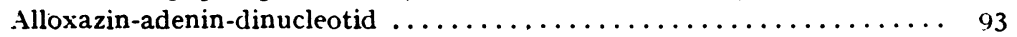

6,7-Dimethyl-9-1-araboflavin-5'-phosphorsäure . . . . . . . . . . 94

Cofermentwirkung der freien Flavine; Konstitutioł und Vitamin $\mathrm{B}_{2}$-Wirkung 95

Literaturverzeichnis.............................. 98 
Seite

Chemistry of the iodine compounds of the thyroid. By Professor C. R. Harington, University College Hospital Medical Schiol, London I03

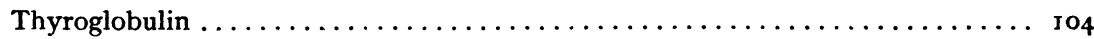

Preparation of thyroglobulin........................ 104

Molecular weight and isoelectric point of thyroglobulin $\ldots \ldots \ldots \ldots \ldots$ ro5

Amino-acid composition of thyroglobulin................... 105

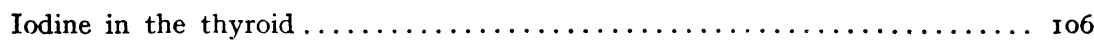

Isolation of iodine compounds from the thyroid $\ldots \ldots \ldots \ldots \ldots \ldots \ldots$ Io 7

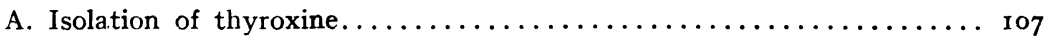

B. Isolation of $3: 5$-diiodotyrosine $\ldots \ldots \ldots \ldots \ldots \ldots \ldots \ldots \ldots \ldots \ldots$ rog

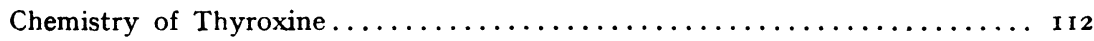

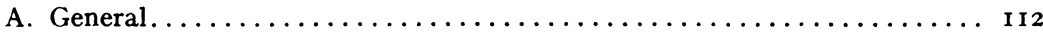

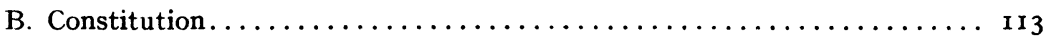

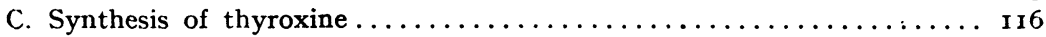

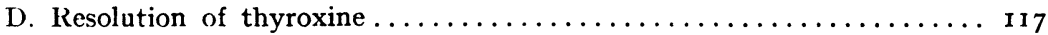

E. Derivatives of dl-thyroxine $\ldots \ldots \ldots \ldots \ldots \ldots \ldots \ldots \ldots \ldots \ldots \ldots \ldots \ldots \ldots \ldots \ldots$

Chemistry of diiodotyrosine. ........................ I19

Relationship of chemical structure to physiological action in the thyroxine series 120

Configurative relationship of thyroxine to tyrosine............... I2I

Mode of combination of thyroxine and diiodotyrosine in the thyroid ...... 122

Quantitative relationship of thyroxine and diiodotyrosine in the thyroid .... 125

Nature of the active secretion of the thyroid $\ldots \ldots \ldots \ldots \ldots \ldots \ldots \ldots$ I 27

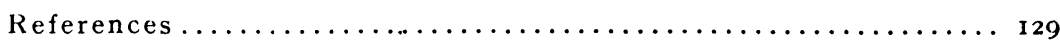

The structure and synthesis of vitamin C (ascorbic acid) and its analogues. By Professor E. L. HIRST, L'niversity of Bristol (England) 132

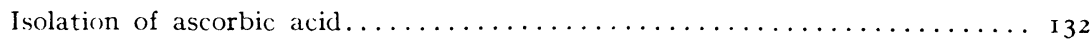

Chemical properties and constitution of ascorbic acid ............ I 34

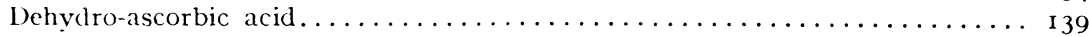

Condensation products of dehydro-ascorbic acid with phenylhydrazine and o-phenylene diamine $\ldots \ldots \ldots \ldots \ldots \ldots \ldots \ldots \ldots \ldots \ldots \ldots \ldots \ldots \ldots \ldots$

Reactions of ascoirbic acid with acetone, triphenylmethyl chloride and p-nitro-

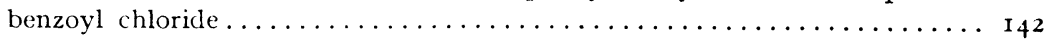

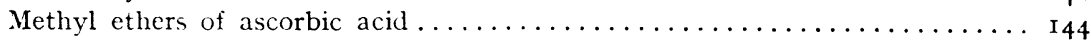

Synthesis of ascorbic acid and its analogues $\ldots \ldots \ldots \ldots \ldots \ldots \ldots \ldots \ldots \ldots$ I47

1. By addition of hydrogen cyanide to osones $\ldots \ldots \ldots \ldots \ldots \ldots \ldots \ldots$ I 47

2. By isomerisation and lactonisation of 2 -keto-acids $\ldots \ldots \ldots \ldots \ldots$ I 49

3. By condensation of sugars with ethyl glyoxylate $\ldots \ldots \ldots \ldots \ldots \ldots$ I 5 I

()ther analogues of ascorbic acid $\ldots \ldots \ldots \ldots \ldots \ldots \ldots \ldots \ldots \ldots \ldots \ldots \ldots \ldots$

I. Derivatives of oxytetronic acid $\ldots \ldots \ldots \ldots \ldots \ldots \ldots \ldots \ldots \ldots \ldots \ldots \ldots$

2. Analogues of ascorbic acid containing six-membered rings ...... I 53

3. Reductic acid and reductone $\ldots \ldots \ldots \ldots \ldots \ldots \ldots \ldots \ldots \ldots \ldots \ldots \ldots$

Intiscorbutic activity of analogues of ascorbic acid ........... I55

References $\ldots \ldots \ldots \ldots \ldots \ldots \ldots \ldots \ldots \ldots \ldots \ldots \ldots \ldots \ldots \ldots \ldots \ldots \ldots \ldots \ldots$ 
Neuere Richtungen der Oligosaccharid-Synthese. Von Professor Dr.

G. ZEMPLÉN, Technische Universität Budapest............... 160

I. Synthesen aus Acetohalogen-Verbindungen mit Hilfe von Silberoxyd oder Silbercarbonat $\ldots \ldots \ldots \ldots \ldots \ldots \ldots \ldots \ldots \ldots \ldots \ldots \ldots \ldots, 160$

Oligosaccharide vom Typus der Trehalose................ I60

Synthesen mit Hilte von Tritylverbindungen $\ldots \ldots \ldots \ldots \ldots \ldots \ldots 163$

Synthesen mit Acetonverbindungen................... I69

Synthese der Cellobiose.......................... 174

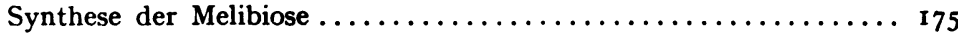

Synthese von methylierten Oligosacchariden ............. I75

II. Quecksilberacetat-Methode $\ldots \ldots \ldots \ldots \ldots \ldots \ldots \ldots \ldots \ldots \ldots \ldots \ldots \ldots \ldots$ I 78

Synthesen mit I-Methyl-2,3,4-triacetyl-glykose $\ldots \ldots \ldots \ldots \ldots \ldots$ I 80 Synthesen mit I-Chlor-2,3,4-triacetyl-glykose und analogen Ver-

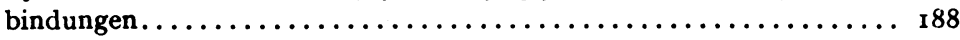

III. Darstellung neuer Oligosaccharide durch Abbau der acetylierten Nitrile I95

IV. Darstellung neuer Oligosaccharide aus Verbindungen vom Typus des Cellobials................................... 199

V. Darstellung neuer Oligosaccharide durch Umlagerung der acetylierten Zucker mit sublimiertem Aluminiumchlorid................. 202

VI. Umlagerung mit Fluorwasserstoff..................... 203

VII. Darstellung neuer Oligosaccharide auf Grund der Umlagerungen nach

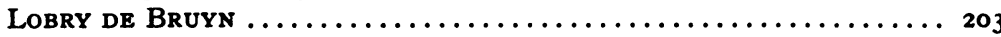

VIII. Synthesen mit dem Brigrschen Anhydrid................ 205

IX. Enzymatische Synthesen......................... 207

Literaturverzeichnis:............................ 207

Chitin und seine Spaltprodukte. Von Professor Dr. L. ZECHMEISTER und Dr. G. Tóth, Universităt Pécs (Ungarn) $\ldots \ldots \ldots \ldots \ldots \ldots \ldots \ldots 2$

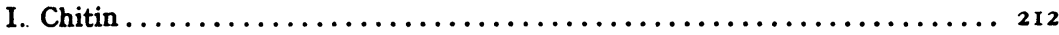

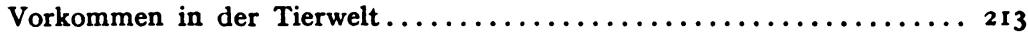

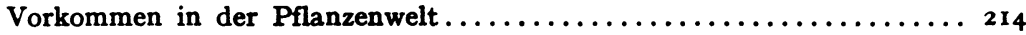

Identităt von pflanzlichem und tierischem Chitin.............. 215

Röntgenographische Untersuchung. Bau der Chitinkette $\ldots \ldots \ldots \ldots \ldots 216$

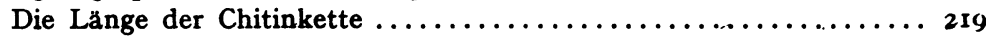

$\beta$-Glucosidische Verknüpfung der Bausteine ................ 220

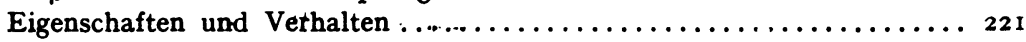

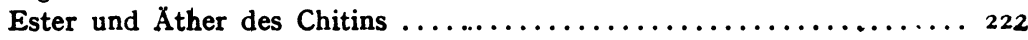

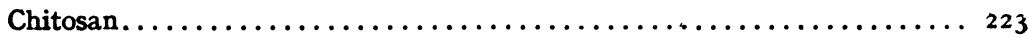

Enzymatischer Abbau des Chitins....................... 224

Abbau des Chitins durch Mikroorganismen.................. 226

II. Die $Z$ wischenprodukte des Chitinabbaues $\ldots \ldots \ldots \ldots \ldots \ldots \ldots \ldots \ldots \ldots 27$

Isolierung. ......................................... 227

Konstitution der Chitobiose und Chitotriose $\ldots \ldots \ldots \ldots \ldots \ldots \ldots \ldots \ldots$, 2209

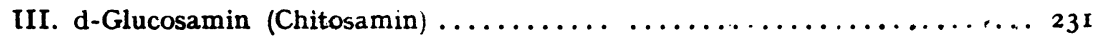

Konfiguration des d-Glucosamins $\ldots \ldots \ldots \ldots \ldots \ldots \ldots \ldots \ldots \ldots \ldots, 233$

a) Optische Methode.............................. 233

b) Komplexchemische Methode $\ldots \ldots \ldots \ldots \ldots \ldots \ldots \ldots \ldots \ldots \ldots, 235$

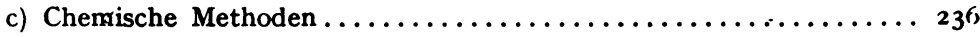

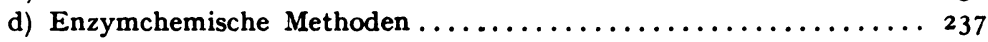


Desaminierung des Glucosamins in Organismen $\ldots \ldots \ldots \ldots \ldots \ldots \ldots 237$

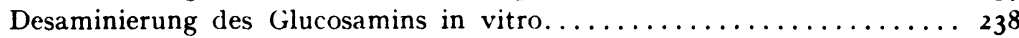

Literaturverzeichnis.........................240

Tabak-alkaloide. Von Professor Dr. E. SPÄTh und Dr. F'. KuffNER,

II. Chemisches Iniversitätslaboratorium Wien ............... 248

Zur Geschichte des Tabaks........................... 248

Entdeckung des Nicotins ............................. 249

Konstitutionsermittlung und Synthese des Nicotins ............... 250

Synthetische Isomere des Nicotins . ...................... 259

Nebenalkaloide des Tabaks (Isolierung, Konstitution) $\ldots \ldots \ldots \ldots \ldots \ldots \ldots$ 26r

Die Basen des Tabakrauebes (Isolierung, Konstitution)............. 276

Synthesen von Tabakrauch- und Tabak-nebenbasen............... 278

Vorkommen von Nicotin, Entstehung in der Pflanze, Wirksamkeit....... 280

Bestimmung des Nicotins . . . . . . . . . . . . . . . . . . . . . 284

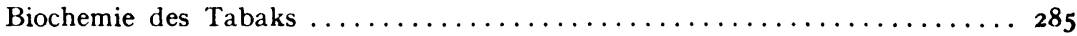

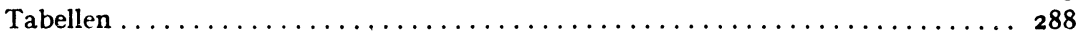

Literaturverzeichnis............................ 292

La spectrochimie de fluorescence dans l'étude des produits bio-

logiques. Par Professeur $\mathrm{CH}_{\mathrm{H}}$. DhéRÉ, Cniversité de Fribourg (Suisse) 30r

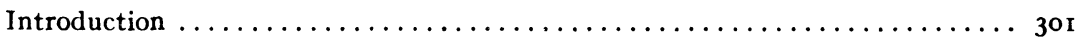

I. Conditions à réaliser pour l'étude des spectres de fluorescence...... 304

II. Examen des spectres de fluorescence .................... 306

III. Principaux spectres de fluorescence ..................... 309

A. Hydrocarbones (glucides) et glucosides .................. 309

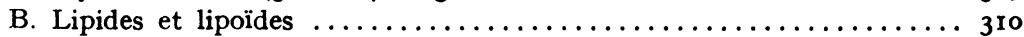

C. Protéines et aminoacides .......................... 3 II

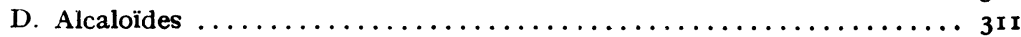

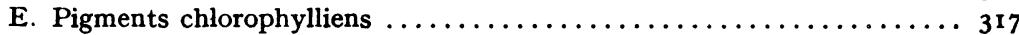

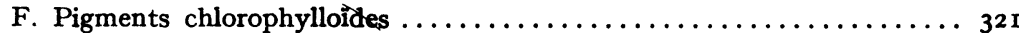

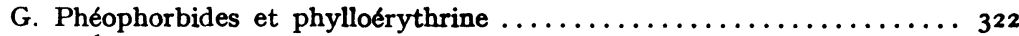

H. Phycochromoprotéides............................... 323

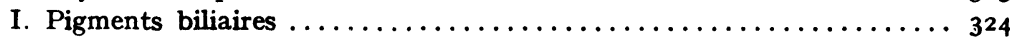

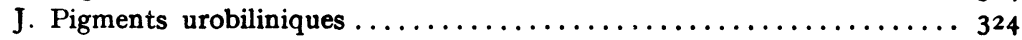

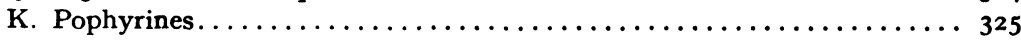

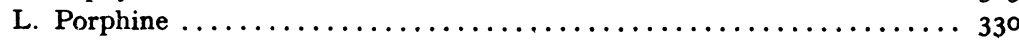

M. Complexes métalliques des pophyrines $\ldots \ldots \ldots \ldots \ldots \ldots \ldots \ldots \ldots 331$

N. Carbures d'hydrogène ............................. 332

O. Pigments caroténoïdes et vitamine A................... $33^{2}$

P. Oxypénicilliopsine et hypéricine...................... 332

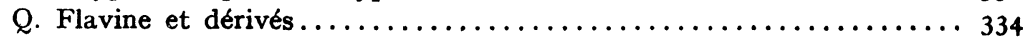

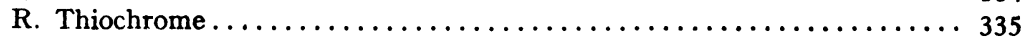

S. Amide de l'acide N-d-glucosido-o-dihydro-nicotique $\ldots \ldots \ldots \ldots \ldots 335$

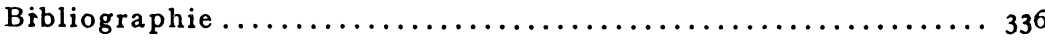

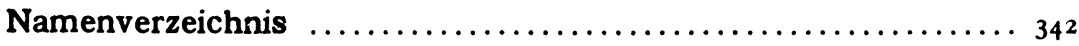

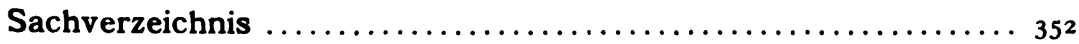

